\title{
ELLIPSOIDAL SETS FOR STATIC OUTPUT FEEDBACK
}

\author{
Dimitri Peaucelle - Denis Arzelier - Regis Bertrand
}

\author{
LAAS - CNRS \\ 7, Avenue du Colonel Roche - 31077 Toulouse cedex - France \\ Tel. 0561336417 fax: 0561336969 \\ email: peaucelle@laas.fr - arzelier@laas.fr-rbertran@laas.fr
}

\begin{abstract}
The static output feedback synthesis for LTI systems is considered. It is shown to have analogies with robust analysis, in particular the existence of an output feedback gain is equivalent to the existence of some quadratic separator. These considerations lead to formulate the problem as the synthesis of an ellipsoidal set of stabilising controllers. Based on this formulation important issues are developed for fragility, restricted controllers and also root-clustering. Copyright (C)2001 IFAC
\end{abstract}

Keywords: Output Feedback, Linear Systems, Matrix Inequalities, Fragility, Root-Clustering, Quadratic Separation.

\section{INTRODUCTION}

The Static Output Feedback (SOF) design is a central problem in control engineering and is still open (Bernstein, 1992; Blondel et al., 1995; Syrmos et al., 1997). In (Fu and Luo, 1997) a formulation of the SOF problem based on the non convex relation $\mathbf{X P}=\mathbb{1}$, is shown to be NP-hard. Some papers propose therefore numerical algorithms with no global guaranteed convergence, (El Ghaoui et al., 1997; El Ghaoui and Niculescu, 2000). Nevertheless, some examples show the nice convergence properties, (de Oliveira and Geromel, 1997), and are satisfactory for some applications. The aim of this paper is to rely on these practical algorithms and discuss the opportunity to solve the SOF problem with various constraints on the closedloop system and on the matrix gain.

As in the papers cited above, we explore the SOF synthesis based on LMI formulations. All synthesis objectives are expressed with LMIs, constrained by a single non convex inequality $\mathbf{X} \leq \mathbf{Y Z}^{-1} \mathbf{Y}^{\prime}$.

The key result is closely related to topological separation (Goh and Safonov, 1995). This framework amounts to tackle the stability of interconnected systems (figure 1) via the existence of a topological separator between the graph of the first system $\left(\Sigma_{1}\right)$ and the inverse graph of the other $\left(\Sigma_{2}\right)$. Based on this framework, major contributions have been made for robust control, (Iwasaki and Hara, 1998; El Ghaoui and Niculescu, 2000; Peaucelle and Arzelier, 2001).

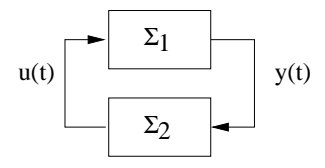

Figure 1. Interconnected systems

For the SOF problem, we consider the separation between the open-loop system and the controller constant matrix gain $\left(\Sigma_{2}=\mathbf{K}\right)$. In this case, (Iwasaki and Hara, 1998) shows that the separator can be chosen without conservatism among real constant matrices. The synthesis of a static output feedback gain is therefore equivalently replaced by the synthesis of some quadratic constant separator that characterises a set of stabilising controllers.

A by-product of the proposed framework is to introduce extra decision variables. These new "slack" variables have an equivalent status as those introduced in (Geromel et al., 1998; de Oliveira et al., 1999; Peaucelle et al., 2000). They create extra degrees of free- 
dom, that may be exploited for non conservative multiobjective and robustness purposes.

The existing SOF results in literature focus mainly on stabilisability and few performance objectives such as $H_{2}$ or $H_{\infty}$ norms are discussed, (Iwasaki, 1999; Gahinet and Apkarian, 1994). The proposed framework may be applied for most of the performance criteria that have analysis convex LMI formulations, (Boyd et al., 1994; Peaucelle, 2000). For conciseness reasons all criteria are not considered in the paper. Only pole location in LMI regions, (Chilali and Gahinet, 1996), is detailed.

In addition, the adopted approach is particularly appropriate for specifying constraints on the controllers. The first issue is the fragility as introduced in (Keel and Bhattacharyya, 1997). Having defined a set of admissible controllers, the stability and performance objectives are assessed whatever some uncertainties on the SOF gain. The second issue concerns structural constraints on the controller. Some non conservative LMI based properties allow to restrict the SOF gain to some bounded set.

The outline of the paper is the following. First, some notations are introduced. The third section is devoted to the central result of the paper. Next, some extensions to fragility and constrained controllers explicit the properties of the new SOF design. The fifth section is devoted to pole location design. Finally, the sixth section exposes some adapted LMI-based algorithms tested on telling examples.

\section{PRELIMINARIES}

Notations are standard. $\mathbb{S}^{n}\left(\mathbb{S}_{+}^{n}\right)$ is the subset of symmetric (positive definite) matrices in $\mathbb{R}^{n \times n}$. $A^{\prime}$ is the transpose of the matrix $A$ and $A^{*}$ is its transpose conjugate. $\mathbb{1}$ and $\mathbb{O}$ are respectively the identity and the zero matrices of appropriate dimensions. For symmetric matrices, $>(\geq)$ is the Loëner partial order, i.e., $A>(\geq) B$ if and only if $A-B$ is positive (semi) definite. ${ }^{\prime} A B$ denotes the symmetric product of matrices: $B^{\prime} A B . \otimes$ is the Kronecker product of matrices.

Throughout this paper, a particular set of matrices is used. By extension of the notion of $\mathbb{R}^{n}$ ellipsoids, this type of set is referred to as matrix ellipsoids of $\mathbb{R}^{m \times p}$.

Definition 1. Given three matrices $X \in \mathbb{S}^{p}, Y \in \mathbb{R}^{p \times m}$ and $Z \in \mathbb{S}^{m}$, the $\{X, Y, Z\}$-ellipsoid of $\mathbb{R}^{m \times p}$ is the set of matrices $\mathbf{K}$ satisfying the following matrix inequalities:

$$
Z>\mathbb{0} \quad\left[\begin{array}{ll}
\mathbb{1} & \mathbf{K}^{\prime}
\end{array}\right]\left[\begin{array}{ll}
X & Y \\
Y^{\prime} & Z
\end{array}\right]\left[\begin{array}{l}
\mathbb{1} \\
\mathbf{K}
\end{array}\right] \leq \mathbb{0}
$$

By definition, $K_{o} \triangleq Z^{-1} Y^{\prime}$ is the centre of the ellipsoid and $R \triangleq K_{o}^{\prime} Z K_{o}-X$ its radius. The inequalities (1) write also as:

$$
Z>\mathbb{0} \quad\left(\mathbf{K}-K_{o}\right)^{\prime} Z\left(\mathbf{K}-K_{o}\right) \leq R
$$

Some properties of these sets are pointed out in the following lemma:

\section{Lemma 1.}

i) The $\{X, Y, Z\}$-ellipsoid is non-empty if and only if the radius $(R \geq 0)$ is positive semi-definite.

ii) A matrix ellipsoid is a compact convex set.

iii) The volume of a matrix $\{X, Y, Z\}$-ellipsoid of $\mathbb{R}^{m \times p}$ is related to the volume of the matrix unit ball by:

$$
\frac{V O L(\{X, Y, Z\}-\text { ellipsoid })}{\operatorname{VOL}(\{-\mathbb{1}, \mathbb{0}, \mathbb{1}\}-\text { ellipsoid })}=\sqrt{\frac{\operatorname{det}(R)^{m}}{\operatorname{det}(Z)^{p}}}
$$

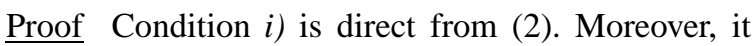
illustrates that the set is closed and bounded. Applying a Schur complement argument, the inequality (1) also has an LMI formulation:

$$
\left[\begin{array}{cc}
X+Y \mathbf{K}+\mathbf{K}^{\prime} Y^{\prime} & \mathbf{K}^{\prime} Z \\
Z \mathbf{K} & -Z
\end{array}\right] \leq 0
$$

which is one way to prove convexity, $i i)$.

The volume is defined in $\mathbb{R}^{m \times p}$ with the Lebesgue measure. Since Lebesgue measure is invariant under translation, the volume of the ellipsoid defined by (2) is the same as for ellipsoid such that $\mathbf{K}^{\prime} Z \mathbf{K} \leq R$. As $Z$ and $R$ are positive definite, take respectively $S_{Z}$ and $S_{R}$ their positive definite square roots (in the particular cases when $R$ is singular, $\operatorname{det}(R)=0$ and the volume is zero; this part of the proof is omitted for conciseness reasons). The last inequality writes as:

$$
\left(S_{Z} \mathbf{K} S_{R}^{-1}\right)^{\prime}\left(S_{Z} \mathbf{K} S_{R}^{-1}\right) \leq \mathbb{1}
$$

Since the linear transformations $K \rightarrow S_{Z} K$ and $K \rightarrow$ $K S_{R}$ multiply Lebesgue measure by $\operatorname{det}\left(S_{Z}\right)^{p}$ and $\operatorname{det}\left(S_{R}\right)^{m}$ respectively, the measure of the ellipsoid is $\operatorname{det}\left(S_{Z}\right)^{-m} \cdot \operatorname{det}\left(S_{R}\right)^{p}$ times the measure of $\mathbf{K}^{\prime} \mathbf{K} \leq \mathbb{1}$ which is the matrix unit sphere and also the set of all linear contractions.

\section{STABILISABILITY OF LTI SYSTEMS}

This section is devoted to the static output feedback (SOF) stabilisability of linear time invariant (LTI), continuous-time systems. Consider such a system with the state-space representation:

$$
\Sigma:\left\{\begin{array}{l}
\dot{x}(t)=A x(t)+B u(t) \\
y(t)=C x(t)+D u(t)
\end{array}\right.
$$

$x \in \mathbb{R}^{n}$ is the state vector, $u \in \mathbb{R}^{m}$ is the input vector and $y \in \mathbb{R}^{p}$ is the output vector. A SOF control law is defined by a constant gain matrix $K$, such that:

$$
u(t)=K y(t)
$$


$\Sigma \star K$ is the closed loop system defined by (6), (7).

Definition 2. The LTI system $\Sigma$ is said to be stabilisable by static output feedback if there exists a matrix gain $\mathbf{K}$ such that $\Sigma \star \mathbf{K}$ is stable.

The stabilisability is tackled in this paper via Lyapunov theory. The stabilisability of the open-loop system is attested by the joint search for a quadratic Lyapunov function $V(x)=x^{\prime} \mathbf{P} x$ and for the SOF gain $\mathbf{K}$.

Theorem 1. The LTI system $\Sigma$ is stabilisable by static output feedback if and only if there exist four matrices $\mathbf{P} \in \mathbb{S}^{n}, \mathbf{X} \in \mathbb{S}^{p}, \mathbf{Y} \in \mathbb{R}^{p \times m}$ and $\mathbf{Z} \in \mathbb{S}^{m}$ that satisfy the following LMI constraints:

$$
\left\{\begin{array}{l}
\mathbf{P}>\mathbb{0} \\
\mathbf{Z}>\mathbb{0} \\
\bullet^{\prime}\left[\begin{array}{ll}
\mathbb{0} & \mathbf{P} \\
\mathbf{P} & \mathbb{0}
\end{array}\right]\left[\begin{array}{ll}
\mathbb{1} & 0 \\
A & B
\end{array}\right]<\bullet^{\prime}\left[\begin{array}{cc}
\mathbf{X} & \mathbf{Y} \\
\mathbf{Y}^{\prime} & \mathbf{Z}
\end{array}\right]\left[\begin{array}{ll}
C & D \\
0 & \mathbb{1}
\end{array}\right]
\end{array}\right.
$$

and simultaneously the non linear constraint:

$$
\mathbf{X} \leq \mathbf{Y Z}^{-1} \mathbf{Y}^{\prime}
$$

Under these conditions a set of stabilising gains is given by the non-empty $\{X, Y, Z\}$-ellipsoid.

Proof of sufficiency Assume conditions (8) and (9) hold for some choice of $P, X, Y$ and $Z$. Choose $V(x)=x^{\prime} P x$ as Lyapunov function. Take any matrix $K$ that belongs to the $\{X, Y, Z\}$-ellipsoid defined by (1). The ellipsoid is non empty due to condition (9) and lemma 1. The elimination lemma (Skelton et al., 1998) applied to condition (1) implies that there exists a strictly positive scalar $\tau>0$ such that:

$$
\left[\begin{array}{ll}
X & Y \\
Y^{\prime} & Z
\end{array}\right] \leq \tau\left[\begin{array}{c}
K^{\prime} \\
-\mathbb{1}
\end{array}\right][K-\mathbb{1}]
$$

For this choice of $K$ and $\tau$, one gets:

$$
\bullet\left[\begin{array}{ll}
0 & P \\
P & \mathbb{O}
\end{array}\right]\left[\begin{array}{ll}
\mathbb{1} & \mathbb{0} \\
A & B
\end{array}\right]<\tau \bullet^{\prime}[K-\mathbb{1}]\left[\begin{array}{ll}
C & D \\
\mathbb{O} & \mathbb{1}
\end{array}\right]
$$

which implies for all non-zero vectors $\left(x^{f} u^{\prime}\right)^{\prime} \neq 0$ :

$$
2 x^{\prime} P(A x+B u)<\tau \bullet^{\prime}(K C x+K D u-u)
$$

Along the trajectories of $\Sigma \star K$ the vectors $x$ and $u$ are such that $\dot{x}=A x+B u$ and $u=K C x+K D u$. The derivative of the Lyapunov function, $\dot{V}(x)$, along the trajectories of the closed-loop system is definite negative. The stability is proved for any matrix gain of the $\{X, Y, Z\}$-ellipsoid.

Proof of necessity Let $K$ be a stabilising gain and $\bar{V}(x)=x^{J} P x$ be a Lyapunov function that proves the stability of $\Sigma \star K$. The inequality $\dot{V}(x)<0$ also writes as:

$$
\left(\begin{array}{l}
x \\
u
\end{array}\right)^{\prime}\left[\begin{array}{ll}
\mathbb{1} & \mathbb{O} \\
A & B
\end{array}\right]^{\prime}\left[\begin{array}{ll}
\mathbb{O} & P \\
P & \mathbb{O}
\end{array}\right]\left[\begin{array}{ll}
\mathbb{1} & \mathbb{0} \\
A & B
\end{array}\right]\left(\begin{array}{l}
x \\
u
\end{array}\right)<0
$$

for all non zero vectors $\left(x^{\prime} u^{\prime}\right)^{\prime} \neq 0$ such that:

$$
[K C K D-\mathbb{1}]\left(\begin{array}{l}
x \\
u
\end{array}\right)=0
$$

or equivalently for all $\left(x^{\prime} u^{\prime}\right)^{\prime} \neq 0$ such that:

$$
\left(\begin{array}{l}
x \\
u
\end{array}\right)^{\prime} \bullet^{\prime}\left[\begin{array}{ll}
K & -\mathbb{1}
\end{array}\right]\left[\begin{array}{ll}
C & D \\
0 & \mathbb{1}
\end{array}\right]\left(\begin{array}{l}
x \\
u
\end{array}\right) \leq \mathbb{0}
$$

Applying the S-procedure (Yakubovitch, 1971), there exists a scalar $\tau>0$ such that (8) with:

$$
X=\tau K^{\prime} K \quad Y=-\tau K^{\prime} \quad Z=\tau \mathbb{1}
$$

This proves the necessity with the strictly satisfied non linear constraint: $X=Y Z^{-1} Y^{\prime}$.

Remark 1. As already discussed in the first section, theorem 1 is closely related to quadratic separation, (Goh and Safonov, 1995; Iwasaki and Hara, 1998). The symmetric matrix composed of $\mathbf{X}, \mathbf{Y}$ and $\mathbf{Z}$ is a topological separator between the graph of the system $\Sigma$ and a stabilising set of controllers. On way to demonstrate this is to apply the Kalman-YakubovichPopov lemma. The inequality (8) implies that for all $\omega \in \mathbb{R} \cup\{\infty\}$ :

$$
\mathbb{0}<\left[\begin{array}{c}
\Sigma(j \omega) \\
\mathbb{1}
\end{array}\right]^{*}\left[\begin{array}{ll}
\mathbf{X} & \mathbf{Y} \\
\mathbf{Y}^{\prime} & \mathbf{Z}
\end{array}\right]\left[\begin{array}{c}
\Sigma(j \omega) \\
\mathbb{1}
\end{array}\right]
$$

where $\Sigma(s)=D+C(s \mathbb{1}-A)^{-1} B$ is the system transfer matrix. Inequalities (1) and (17) compose the separation result. It demonstrates that the quadratic separator proves simultaneously the stabilisability with respect to a whole set of controllers.

\section{SOME RELATED ISSUES}

\subsection{Fragility issues}

An important issue of theorem 1 concerns the fragility of the control law. By extension of robust stability we define the notion of resilience:

Definition 3. Let $K_{o}$ be a stabilising gain for the system $\Sigma$ and let $\Delta_{K}$ be a set of additive uncertainties on the control law $K_{o}$. The controller is said to be fragile to $\Delta_{K}$ if for some $\Delta_{K} \in \Delta_{K}$ the closed loop $\Sigma \star K$ with $K=K_{o}+\Delta_{K}$, is unstable. On the contrary, the controller is said to be non-fragile, (Yang and Wang, 2001), or resilient, (Corrado and Haddad, 1999), if the closed-loop is stable for all uncertainties $\Delta_{K} \in \Delta_{K}$.

Resilience has the same status as robustness. The latter notion concerns the properties of the closed loop system with respect to misknowings on the system 
model, while the former deals with possible uncertainties that may occur when implementing the computed controller model.

Corollary 1. Assume the matrices $P, X, Y$ and $Z$ satisfy the constraints (8) and (9). The central controller $K_{o}$ is resilient to all additive uncertainty $\Delta_{K}$ such that:

$$
K=K_{o}+\Delta_{K} \quad \Delta_{K}^{\prime} Z \Delta_{K} \leq R
$$

Proof The proof is direct since a single Lyapunov function $V(x)=x^{\prime} P x$ proves the stability of all closedloop systems. The fragility issues are a by-product of the quadratic separation as discussed in remark 1.

The result of corollary 1 illustrates the fact that the ellipsoidal output feedback design is appropriate to deal with fragility. It gives an admissible set of uncertainties at the end of the design procedure. The volume (see lemma 1) of the resulting $\{X, Y, Z\}$-ellipsoid can be used to compare SOF sets and appreciate their respective resilience. Unfortunately, the volume does not take the geometry into account. When a particular geometry is wanted, the two following corollaries show how to modify the initial matrix inequality problem.

Corollary 2. Assume the matrices $P, X, Y$ and $Z$ satisfy (8) with the constraints:

$$
\mathbf{Z}=\mathbb{1} \quad 0<\rho \mathbb{1} \leq \mathbf{Y} \mathbf{Y}^{\prime}-\mathbf{X}
$$

then the central controller $K_{o}=-Y^{\prime}$ is resilient to all additive norm bounded uncertainty such that:

$$
K=K_{o}+\Delta_{K} \quad \Delta_{K}^{\prime} \Delta_{K} \leq \rho \mathbb{1}
$$

Corollary 3. Assume the matrices $P, X, Y$ and $Z$ satisfy (8) with the constraint:

$$
\mathbf{X} \leq\left(1-\bar{\delta}^{2}\right) \mathbf{Y} \mathbf{Z}^{-1} \mathbf{Y}^{\prime}
$$

then the central controller $K_{o}=-Z^{-1} Y^{\prime}$ is resilient to all multiplicative uncertainty, (Corrado and Haddad, 1999; Yang and Wang, 2001), such that:

$$
K=K_{o}+\delta K_{o} \quad|\delta| \leq \bar{\delta}
$$

\subsection{Bounded controller issues}

For implementation purposes, it is important for some applications to look for controllers with bounded gains. Controllers with high amplification gains are quite difficult to implement in practice. Therefore, consider the constraint on the norm of the controller:

$$
\mathbf{K}^{\prime} \mathbf{K} \leq \rho_{K} \mathbb{1}
$$

where $\sqrt{\rho_{K}}$ bounds the controller's norm. Equivalently, it corresponds to look for controllers belonging to the $\left\{-\rho_{K} \mathbb{1}, \mathbb{0}, \mathbb{1}\right\}$-ellipsoid.
As a generalisation, consider restrictions such as:

Find a stabilising gain $\mathbf{K}$ belonging to a specified $\left\{X_{K}, Y_{K}, Z_{K}\right\}$-ellipsoid

In this paper's framework, such a specification amounts to find a stabilising $\{\mathbf{X}, \mathbf{Y}, \mathbf{Z}\}$-ellipsoid included in the specified $\left\{X_{K}, Y_{K}, Z_{K}\right\}$-ellipsoid. The resulting synthesis problem with ellipsoidal constraints on the feedback gain can then be solved as follows.

Theorem 2. If there exist four matrices $\mathbf{P} \in \mathbb{S}^{n}, \mathbf{X} \in$ $\mathbb{S}^{p}, \mathbf{Y} \in \mathbb{R}^{p \times m}, \mathbf{Z} \in \mathbb{S}^{m}$ and a scalar $\mathbf{v}$ that satisfy simultaneously the constraints (8), (9) and the LMI constraint:

$$
\left\{\begin{array}{l}
\mathbf{v}>0 \\
\mathbf{v}\left[\begin{array}{ll}
X_{K} & Y_{K} \\
Y_{K}^{\prime} & Z_{K}
\end{array}\right] \leq\left[\begin{array}{ll}
\mathbf{X} & \mathbf{Y} \\
\mathbf{Y}^{\prime} & \mathbf{Z}
\end{array}\right]
\end{array}\right.
$$

then the non-empty matrix $\{X, Y, Z\}$-ellipsoid is a subset of the $\left\{X_{K}, Y_{K}, Z_{K}\right\}$-ellipsoid and each of its elements is stabilising for the LTI system $\Sigma$.

Proof Conditions (8), (9) ensure that the $\{X, Y$, $Z$ \}-ellipsoid is a set of stabilising controllers due to theorem 1. Moreover, condition (24) writes for all gains $K$ of the $\{X, Y, Y\}$-ellipsoid as, $v>0$, and:

$$
\vee \bullet^{\prime}\left[\begin{array}{ll}
X_{K} & Y_{K} \\
Y_{K}^{\prime} & Z_{K}
\end{array}\right]\left[\begin{array}{l}
\mathbb{1} \\
K
\end{array}\right] \leq \bullet^{\prime}\left[\begin{array}{ll}
X & Y \\
Y^{\prime} & Z
\end{array}\right]\left[\begin{array}{l}
\mathbb{1} \\
K
\end{array}\right] \leq \mathbb{0}(25)
$$

$K$ belongs to the $\left\{X_{K}, Y_{K}, Z_{K}\right\}$-ellipsoid.

\section{POLE LOCATION}

Finding easy testable conditions for a dynamical system to have all its poles in a sub-region of the complex plane, has been thoroughly investigated in the last years. A major result is proposed in (Chilali and Gahinet, 1996). It extends Lyapunov matrix inequalities for a new class of convex subregions named LMI regions. We focus on this type of regions, with a revisited formalism presented in (Peaucelle et al., 2000).

Definition 4. Let $X_{R}, Y_{R}$ and $Z_{R}$ be square complex valued matrices of same dimensions $\left(\in \mathbb{C}^{d \times d}\right)$ and assume that:

$$
\left[\begin{array}{ll}
X_{R} & Y_{R} \\
Y_{R}^{*} & Z_{R}
\end{array}\right]=\left[\begin{array}{ll}
X_{R} & Y_{R} \\
Y_{R}^{*} & Z_{R}
\end{array}\right]^{*} \quad Z_{R} \geq 0
$$

The $\left\{X_{R}, Y_{R}, Z_{R}\right\}$-region of the complex plane is the convex open set of complex scalars such that:

$$
\left\{s \in \mathbb{C}: X_{R}+s Y_{R}+s^{*} Y_{R}^{*}+s s^{*} Z_{R}<\mathbb{0}\right\}
$$

Examples of the most commonly used $\left\{X_{R}, Y_{R}, Z_{R}\right\}$ regions are all of order $d=1$ and correspond to usual specifications on stability, decay rate or oscillation 
damping. Regions of order greater than $d=1$ are mainly used for specifying intersections of lower order regions, (Chilali and Gahinet, 1996).

Definition 5. An LTI system is said to be $\left\{X_{R}, Y_{R}\right.$, $\left.Z_{R}\right\}$-stable if all its poles belong to the $\left\{X_{R}, Y_{R}, Z_{R}\right\}$ region. It is said to be $\left\{X_{R}, Y_{R}, Z_{R}\right\}$-stabilisable by static output feedback if there exists a matrix gain $\mathbf{K}$ such that $\Sigma \star \mathbf{K}$ is $\left\{X_{R}, Y_{R}, Z_{R}\right\}$-stable.

Note that the definition of $\left\{X_{R}, Y_{R}, Z_{R}\right\}$-stability includes the usual stability of continuous time $(\{0,1,0\}$ region) and discrete time systems $(\{-1,0,1\}$-region).

Theorem 3. The LTI system $\Sigma$ is $\left\{X_{R}, Y_{R}, Z_{R}\right\}$ stabilisable by static output feedback if and only if there exist four matrices $\mathbf{P} \in \mathbb{S}^{n}, \mathbf{X} \in \mathbb{S}^{p}, \mathbf{Y} \in \mathbb{R}^{p \times m}$ and $\mathbf{Z} \in \mathbb{S}^{m}$ that satisfy the non linear constraint (9) and simultaneously the following LMI constraints:

$$
\left\{\begin{array}{rl}
\mathbf{P}>\mathbb{0} & \mathbf{Z}>\mathbb{0} \\
\bullet^{\prime}\left[\begin{array}{cc}
X_{R} \otimes \mathbf{P} & Y_{R} \otimes \mathbf{P} \\
Y_{R}^{\prime} \otimes \mathbf{P} & Z_{R} \otimes \mathbf{P}
\end{array}\right]\left[\begin{array}{ccc}
\mathbb{1} & 0 \\
\mathbb{1} \otimes A & \mathbb{1} \otimes B
\end{array}\right] \\
\quad<\bullet^{\prime}\left[\begin{array}{cc}
\mathbb{1} \otimes \mathbf{X} & \mathbb{1} \otimes \mathbf{Y} \\
\mathbb{1} \otimes \mathbf{Y}^{\prime} & \mathbb{1} \otimes \mathbf{Z}
\end{array}\right]\left[\begin{array}{ccc}
\mathbb{1} \otimes C & \mathbb{1} \otimes D \\
0 & \mathbb{1}
\end{array}\right]
\end{array}\right.
$$

The $\{X, Y, Z\}$-ellipsoid is then a set of $\left\{X_{R}, Y_{R}, Z_{R}\right\}$ stabilising gains.

Proof The major part is omitted. It follows the proof of theorem 1 and is based on pole location Lyapunovlike results, (Chilali and Gahinet, 1996).

\section{AN LMI-BASED ALGORITHM AND EXAMPLES}

The numerical examples are solved using a first order iterative algorithm called the cone complementarity linearisation algorithm, (El Ghaoui et al., 1997). Its properties are not reminded here. Just note that the algorithm has no guaranteed convergence but works nicely on the examples we tested.

To tackle the specificities of the non linear constraint of this paper an unknown matrix is added, $\hat{\mathbf{X}}$. Associated to this matrix the following inequalities are added:

$$
\mathbf{X} \leq\left(1-\bar{\delta}^{2}\right) \hat{\mathbf{X}} \quad\left[\begin{array}{ll}
\hat{\mathbf{X}} & \mathbf{Y} \\
\mathbf{Y}^{\prime} & \mathbf{Z}
\end{array}\right] \geq \mathbb{0}
$$

and the algorithm is used to make $\hat{\mathbf{X}}$ converge towards $\mathbf{Y}^{\prime} \mathbf{Z}^{-1} \mathbf{Y}$. The algorithm is stopped as soon as (21) is satisfied. Note that the exact convergence of $\hat{\mathbf{X}}$ is not required. If $\bar{\delta}=0$ the stopping criteria is the non-emptiness of the $\{X, Y, Z\}$-ellipsoid. Otherwise, $(\bar{\delta} \neq 0)$ the resilience regarding to multiplicative uncertainty is assessed.
All following tests were performed using the solver (Sturm, 1999). Less than 10 iterations of the algorithm where needed and the computation time was about $10 \mathrm{~s}$ on a Sun Ultra 5 computer.

This algorithm has been tested for the VTOL helicopter model, (Iwasaki, 1999). First, fragility with respect to multiplicative uncertainty is considered without any other constraints. For $\bar{\delta}=0,0.25$ and 0.5 , three different $\{X, Y, Z\}$-ellipsoids are obtained. These ellipsoids are plotted in figure 2 where $k_{1}$ and $k_{2}$ are respectively the two components of the matrix gain $\mathbf{K}$.

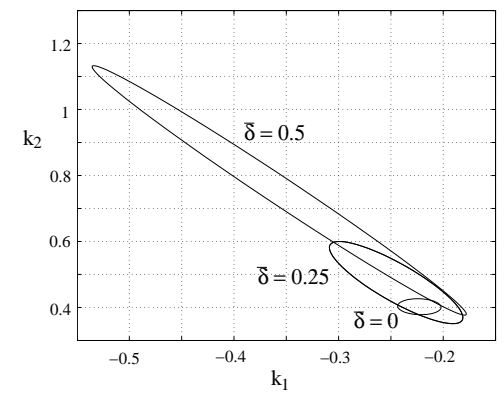

Figure 2. SOF ellipsoids

The interior of each ellipsoid is a set of SOF stabilising gains. Among these, the centre of each ellipsoid is assessed to be a non fragile controller as long as the uncertain gain $K_{o}+\Delta K$ does not exit the ellipsoid. As prescribed by corollary 3 , the "size" of the ellipsoids grows with $\bar{\delta}$. In this example, their volume is respectively $5 \cdot 10^{-4}, 4 \cdot 10^{-3}$ and $9 \cdot 10^{-3}$ time the volume of the unit ball. The less fragile controller, $K_{o}=\left[\begin{array}{lll}-0.3567 & 0.7547\end{array}\right]^{\prime}$, is the centre of the ellipsoid found for $\bar{\delta}=0.5$.

In the second experimentation an additional pole location constraint and an additional ellipsoidal restriction on the controller are considered such that:

$$
\begin{array}{lll}
X_{R}=0.3 & Y_{R}=1 & Z_{R}=0 \\
X_{K}=100 & Y_{K}=[-10-10] & Z_{K}=\mathbb{1}_{2}
\end{array}
$$

The pole location constraint imposes the poles to have a real part less than -0.15 and the ellipsoidal constraint on the controller is a disk (plotted in dashed line on figure 3). For each resilience specification the algorithm converges to different $\{X, Y, Z\}$-ellipsoid that are plotted on figure 3 .

Here again the volume of the SOF ellipsoids is augmented when taking $\bar{\delta}$ large. All controllers included in these ellipsoids are stabilising gains. Moreover, they all belong to the $\left\{X_{K}, Y_{K}, Z_{K}\right\}$-ellipsoid and they ensure the closed-loop system to have all its poles in the $\left\{X_{R}, Y_{R}, Z_{R}\right\}$-region. In terms of fragility, the best SOF ellipsoid is found for $\bar{\delta}=0.5$. Its volume is 2 times the volume of the unit ball and its centre is $K_{o}=\left[\begin{array}{ll}2.2307 & 10.9023\end{array}\right]^{\prime}$. Computing the poles of all closed-loop systems such that (22) with $\bar{\delta}=0.5$, one gets that all the poles have their real part less than 0.2. The pole location constraint is strictly satisfied. 


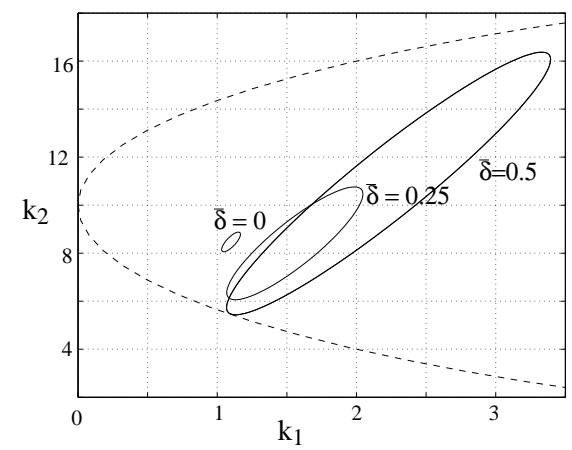

Figure 3. SOF ellipsoids

\section{CONCLUSIONS}

This paper exposes a new approach of static output feedback design. It is proved to have important issues in particular for non-fragile and restrained controller design. Moreover, it is promising for synthesis with performance criteria and a future paper will deal with robust multi-objective design. Another prospective point is to build algorithms particularly dedicated to deal with the non linear constraint.

\section{REFERENCES}

Bernstein, D. (1992). Some open problems in matrix theory arising in linear systems and control. Linear Algebra Applications 162-164, 409-432.

Blondel, V., M. Gevers and A. Lindquist (1995). Survey on the state of systems and control. European J. of Control 1, 5-23.

Boyd, S., L. El Ghaoui, E. Feron and V. Balakrishnan (1994). Linear Matrix Inequalities in System and Control Theory. SIAM Studies in Applied Mathematics. Philadelphia.

Chilali, M. and P. Gahinet (1996). $H_{\infty}$ design with pole placement constraints: An LMI approach. IEEE Trans. on Automat. Control 41, 358-367.

Corrado, J.R. and W.M. Haddad (1999). Static output feedback controllers for systems with parametric uncertainty and controller gain variations. In: American Control Conference. San Diego. pp. 915-919.

de Oliveira, M.C. and J.C. Geromel (1997). Numerical comparison of output feedback design methods. In: American Control Conference. Albuquerque, New Mexico.

de Oliveira, M.C., J. Bernussou and J.C. Geromel (1999). A new discrete-time stability condition. Systems \& Control Letters 37(4), 261-265.

El Ghaoui, L. and Niculescu, S.-I., Eds.) (2000). Advances in Linear Matrix Inequality Methods in Control. Advances in Design and Control. SIAM. Philadelphia.

El Ghaoui, L., F. Oustry and M. AitRami (1997). A cone complementarity linearization algorithm for static ouput-feedback and related problems. IEEE Trans. on Automat. Control 42(8), 11711176.
Fu, M. and Z.-Q. Luo (1997). Computational complexity of a problem arising in fixed order output feedback design. Systems \& Control Letters 30, 209-215.

Gahinet, P. and P Apkarian (1994). A linear matrix inequality approach to $H_{\infty}$ control. Int. J. of Robust and Nonlinear Control 4, 421-448.

Geromel, J.C., M.C. de Oliveira and L. Hsu (1998). LMI characterization of structural and robust stability. Linear Algebra and its Applications 285, 68-80.

Goh, K.C. and M.G. Safonov (1995). Robust analysis, sector and quadratic functionals. In: 34th Conference on Decision and Control. New Orleans, LA.

Iwasaki, T. (1999). The dual iteration for fixedorder control. IEEE Trans. on Automat. Control 44(4), 783-788.

Iwasaki, T. and S. Hara (1998). Well-posedness of feedback systems: Insights into exact robustness analysis and approximate computations. IEEE Trans. on Automat. Control 43(5), 619-630.

Keel, L.H. and S.P. Bhattacharyya (1997). Robust, fragile, or optimal?. IEEE Trans. on Automat. Control 42(8), 1098-1105.

Peaucelle, D. (2000). Formulation Générique de Problèèmes en Analyse et Commande Robuste par les Fonctions de Lyapunov Dépendant des Paramètres. PhD thesis. Université Toulouse III - Paul Sabatier. France.

Peaucelle, D. and D Arzelier (2001). Robust performance analysis with LMI-based methods for real parametric uncertainty via parameter-dependent Lyapunov functions. IEEE Trans. on Automat. Control 46(4), 624-630.

Peaucelle, D., D. Arzelier, O. Bachelier and J. Bernussou (2000). A new robust D-stability condition for real convex polytopic uncertainty. Systems \& Control Letters 40(1), 21-30.

Skelton, R.E., T. Iwazaki and K. Grigoriadis (1998). A unified Approach to Linear Control Design. Taylor and Francis series in Systems and Control.

Sturm, J.F. (1999). Using SeDuMi 1.02, a MATLAB toolbox for optimization over symmetric cones. Optimization Methods and Software 1112, 625-653. URL:http://fewcal.kub.nl/ sturm/software/sedumi.html.

Syrmos, V.L., C.T. Abdallah, P. Dorato and K. Grigoriadis (1997). Static output feedback: A survey. Automatica 33(2), 125-137.

Yakubovitch, V.A. (1971). The S-procedure in nonlinear control theory. Vestnik Leningrad University pp. 62-77.

Yang, G.-H. and J.L. Wang (2001). Non-fragile $H_{\infty}$ control for linear systems with multiplicative controller gain variations. Automatica 37, 727737. 